ARTICLE

https://doi.org/10.1038/s41467-018-08254-y

\title{
Topological unification of time-reversal and particle-hole symmetries in non-Hermitian physics
}

Kohei Kawabata ${ }^{1}$, Sho Higashikawa', Zongping Gong ${ }^{1}$, Yuto Ashida ${ }^{1} \&$ Masahito Ueda ${ }^{1,2}$

Topological phases are enriched in non-equilibrium open systems effectively described by non-Hermitian Hamiltonians. While several properties unique to non-Hermitian topological systems were uncovered, the fundamental role of symmetry in non-Hermitian physics has yet to be fully understood, and it has remained unclear how symmetry protects non-Hermitian topological phases. Here we show that two fundamental anti-unitary symmetries, timereversal and particle-hole symmetries, are topologically equivalent in the complex energy plane and hence unified in non-Hermitian physics. A striking consequence of this symmetry unification is the emergence of unique non-equilibrium topological phases that have no counterparts in Hermitian systems. We illustrate this by presenting a non-Hermitian counterpart of the Majorana chain in an insulator with time-reversal symmetry and that of the quantum spin Hall insulator in a superconductor with particle-hole symmetry. Our work establishes a fundamental symmetry principle in non-Hermitian physics and paves the way towards a unified framework for non-equilibrium topological phases.

\footnotetext{
${ }^{1}$ Department of Physics, University of Tokyo, 7-3-1 Hongo, Bunkyo-ku, Tokyo 113-0033, Japan. ${ }^{2}$ RIKEN Center for Emergent Matter Science (CEMS), Wako, Saitama 351-0198, Japan. Correspondence and requests for materials should be addressed to K.K. (email: kawabata@cat.phys.s.u-tokyo.ac.jp)
} 
$\mathrm{t}$ was Wigner who showed that all symmetries are either unitary or anti-unitary and identified the fundamental role of time-reversal symmetry in anti-unitary operations ${ }^{1}$. Timereversal symmetry is complemented by particle-hole and chiral symmetries, culminating in the Altland-Zirnbauer (AZ) ten-fold classification $^{2}$. The AZ classification plays a key role in characterizing the topological phases ${ }^{3-5}$ of condensed matter such as insulators $^{6-12}$ and superconductors ${ }^{13-16}$, as well as photonic systems ${ }^{17}$ and ultracold atoms ${ }^{18}$, all of which are classified into the periodic table $\mathrm{e}^{19-22}$. Whereas the topological phase in the quantum Hall insulator is free from any symmetry constraint and breaks down in the presence of time-reversal symmetry ${ }^{6,7}$, certain topological phases are protected by symmetry; for example, the quantum spin Hall insulator is protected by time-reversal symmetry $^{8-11}$ and the Majorana chain is protected by particle-hole symmetry ${ }^{14}$.

Despite its enormous success, the existing framework for topological phases mainly concerns equilibrium closed systems. Meanwhile, there has been growing interest in non-equilibrium open topological systems, especially non-Hermitian topological systems $^{23-42}$. In general, non-Hermiticity arises from the presence of energy or particle exchanges with an environment ${ }^{43,44}$, and a number of phenomena and functionalities unique to nonconservative systems have been theoretically predicted ${ }^{45-56}$ and experimentally observed ${ }^{57-67}$. Here symmetry again plays a key role; for example, spectra of non-Hermitian Hamiltonians can be entirely real in the presence of parity-time symmetry ${ }^{46}$. Recently, a topological band theory for non-Hermitian Hamiltonians was developed, and the topological phase in the quantum Hall insulator was shown to persist even in the presence of nonHermiticity $^{31}$. Moreover, topological lasers were proposed and realized on the basis of the interplay between non-Hermiticity and topology $39,41,42$. However, it is yet to be understood how symmetry constrains non-Hermitian systems in general and how symmetry protects non-Hermitian topological phases.

Here we point out that two fundamental anti-unitary symmetries, time-reversal symmetry and particle-hole symmetry, are the two sides of the same symmetry in non-Hermitian physics. In fact, once we lift the Hermiticity constraint on the Hamiltonian $H$, the Wigner theorem dictates that an anti-unitary operator $\mathcal{A}$ is only required to satisfy

$$
\mathcal{A} H \mathcal{A}^{-1}=e^{\mathrm{i} \varphi} H \quad(0 \leq \varphi<2 \pi) .
$$

This suggests that time-reversal symmetry $(\varphi=0)$ and particlehole symmetry $(\varphi=\pi)$ can be continuously transformed into each other in the complex energy plane. This topological unification leads to striking predictions about topological phenomena. In particular, properties intrinsic to topological insulators can appear also in the corresponding topological superconductors, and vice versa: a counterpart of the Majorana chain in a non-Hermitian insulator with time-reversal symmetry and that of the quantum spin Hall insulator in a non-Hermitian superconductor with particle-hole symmetry. We emphasize that such topological phases are absent in Hermitian systems; nonHermiticity alters the topological classification in a fundamental manner, and non-equilibrium topological phases unique to nonHermitian systems emerge as a result of the topological unification of time-reversal and particle-hole symmetries.

\section{Results}

Symmetries and complex spectra. To go beyond the Hermitian paradigm, it is necessary to revisit some fundamental concepts relevant to topology. We start by defining a gapped complex band. Let us consider a complex-band structure $\left\{E_{n}(\boldsymbol{k}) \in \mathbb{C}\right\}$, where $\boldsymbol{k}$ is a crystal wavevector in the Brillouin zone and $n$ is a band index. Since a band gap should refer to an energy range in which no states exist, it is reasonable to define a band $n$ to be gapped such that $E_{m}(\boldsymbol{k}) \neq E_{n}(\boldsymbol{k})$ for all the band indices $m \neq n$ and wavevectors $\boldsymbol{k}$ (Fig. 1) ${ }^{31}$, which is a natural generalization of the gapped band structure in the Hermitian band theory and explains the experimentally observed topological edge states in non-Hermitian systems ${ }^{36-39,41,42}$. Notably, the presence of a complex gap has a significant influence on the non-equilibrium wave dynamics (see Supplementary Note 1 for details). This definition of a complex gap is distinct from that adopted in ref. 68 and hence the corresponding topological classification is different.

We next consider the constraints on complex spectra imposed by anti-unitary symmetry (Table 1 ). A Hamiltonian $H$ has timereversal and particle-hole symmetries if and only if there exist anti-unitary operators $\mathcal{T}$ and $\mathcal{C}$ such that

$$
\mathcal{T} H \mathcal{T}^{-1}=H, \quad \mathcal{C H C}^{-1}=-H,
$$

and $\mathcal{T} z \mathcal{T}^{-1}=z^{*}, \mathcal{C} z \mathcal{C}^{-1}=z^{*}$ for all $z \in \mathbb{C}$. For Hermitian Hamiltonians with entirely real spectra, time-reversal symmetry places no constraints on the real spectra and particle-hole symmetry renders the real spectra symmetric about zero energy. By contrast, for non-Hermitian Hamiltonians, of which spectra are not restricted to be real, time-reversal symmetry renders the spectra symmetric about the real axis ${ }^{46}$, while particle-hole symmetry makes the spectra symmetric about the imaginary axis $^{26,33,52}$; they are topologically equivalent in the complex energy plane (see Supplementary Note 2 for details). This crucial observation leads to the expectation that non-Hermiticity topologically unifies symmetry classes (Fig. 2), as shown below. We note that the role of chiral symmetry is unchanged in nonHermitian physics, since it is defined to be unitary and does not involve complex conjugation.
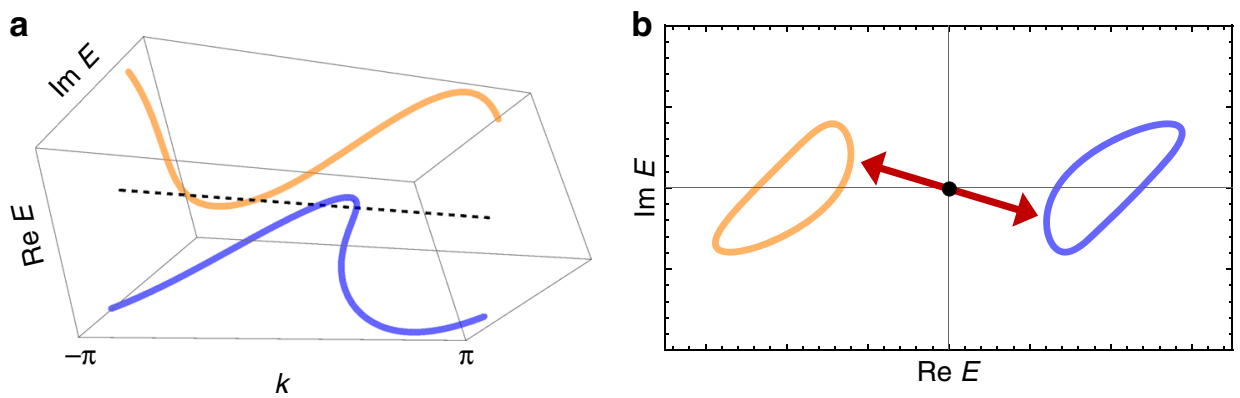

Fig. 1 Gapped band structure for a non-Hermitian Hamiltonian. Energy dispersion for two bands (blue and orange curves) in one dimension: a ( $k$, Re $E$, Im $E$ ) with wavenumber $k$ and complex energy $E$ and $\mathbf{b}$ its projection on the complex energy plane $(\operatorname{Re} E, \operatorname{Im} E)$. The two bands neither touch nor intersect for any $k$, and therefore they are gapped. This definition does not distinguish between real and imaginary parts of energy 
Table 1 Constraints on the complex spectra imposed by the Altland-Zirnbauer (AZ) symmetry

\begin{tabular}{lll} 
AZ symmetry & Hermitian & Non-Hermitian \\
\hline Time-reversal & No constraints & $E \in \mathbb{R}$ or $\left(E, E^{\star}\right)$ \\
Particle-hole & $E=0$ or $(E,-E)$ & $E \in i \mathbb{R}$ or $\left(E,-E^{*}\right)$ \\
Chiral & $E=0$ or $(E,-E)$ & $E=0$ or $(E,-E)$
\end{tabular}

In Hermitian systems, time-reversal symmetry places no constraints on the real spectra, while particle-hole symmetry gives zero energy $E=0$ or opposite-sign pairs $(E,-E)$. In non-Hermitian systems, by contrast, time-reversal symmetry gives real energies $E \in \mathbb{R}$ or complex-conjugate pairs $\left(E, E^{*}\right)$, while particle-hole symmetry gives pure imaginary energies $E \in i \mathbb{R}$ or pairs $\left(E_{,}-E^{*}\right)$. Chiral symmetry gives $E=0$ or pairs $(E,-E)$ in both Hermitian and non-Hermitian systems.

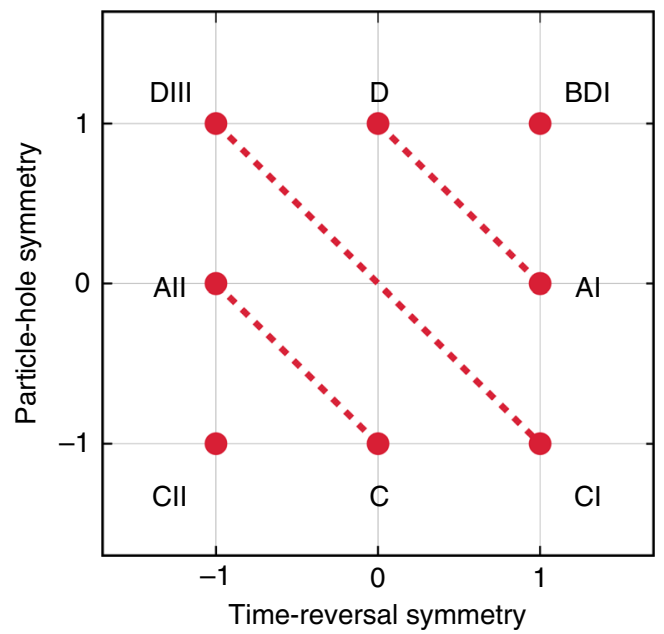

Fig. 2 Altland-Zirnbauer symmetry classes that involve the anti-unitary symmetry (real class). The classes are specified by the values of $\mathcal{T}^{2}= \pm 1$ and $\mathcal{C}^{2}= \pm 1$, and the absence of symmetry is indicated by 0 . The class symbol follows Cartan's notation. While there are eight symmetry classes in Hermitian physics, the classes connected by dotted lines are topologically equivalent and thus the symmetry classes reduce to five in non-Hermitian physics. For instance, a non-Hermitian Hamiltonian having time-reversal symmetry with $\mathcal{T}^{2}=+1$ alone (class $\mathrm{Al}$ ) and that having particle-hole symmetry with $\mathcal{C}^{2}=+1$ alone (class $\mathrm{D}$ ) are equivalent and hence unified

Topological unification. Motivated by the topological equivalence of time-reversal and particle-hole symmetries in the complex energy plane, we consider a general anti-unitary symmetry $\mathcal{A}$ defined by Eq. (1). Here $\mathcal{A}$ reduces to the operator that corresponds to time-reversal (particle-hole) symmetry for $\varphi=0$ $(\varphi=\pi)$. Remarkably, only time-reversal and particle-hole symmetries are allowed when $H$ is Hermitian. To see this, we take Hermitian conjugation of Eq. (1) and use Hermiticity of $H$ $\left(H^{\dagger}=H\right)$ and the definition of anti-unitary symmetry $\left(\mathcal{A}^{\dagger}=\mathcal{A}^{-1}\right)$. We then obtain $\mathcal{A} H \mathcal{A}^{-1}=e^{-\mathrm{i} \varphi} H$, which leads to $\varphi$ $=0, \pi$. For non-Hermitian $H$, on the other hand, there are no such constraints.

We study the continuous deformations between a system with time-reversal symmetry and a system with particle-hole symmetry in the presence of a complex-energy gap and an anti-unitary symmetry $\mathcal{A}$. Such deformations cannot be performed for Hermitian Hamiltonians since only the discrete values $\varphi=0, \pi$ are allowed due to Hermiticity; a topological phase with timereversal symmetry and that with particle-hole symmetry are distinguished in Hermitian physics. Surprisingly, an arbitrary non-Hermitian Hamiltonian $H_{0}$ with time-reversal symmetry can be continuously deformed into a Hamiltonian with particle-hole symmetry because $H_{\varphi}:=e^{-i \varphi / 2} H_{0}$ preserves both complex gap and anti-unitary symmetry $\mathcal{A}$ for all $\varphi$, and $H_{\pi}$ has particle-hole symmetry. Therefore, a topological phase with time-reversal symmetry and that with particle-hole symmetry are unified into the same topological class in non-Hermitian physics. The topological unification of anti-unitary symmetries presents a general symmetry principle in non-Hermitian physics that holds regardless of the definition of a complex gap ${ }^{68}$.

Topological insulator induced by non-Hermiticity. As a consequence of the topological unification of time-reversal and particle-hole symmetries, unique non-Hermitian topological phases emerge that are absent in Hermitian systems. In particular, in accordance with the topological phase in the Majorana chain $(1 \mathrm{D} \text { class } \mathrm{D})^{14}$, non-Hermiticity induces topological phases in one-dimensional insulators that respect time-reversal symmetry with $\mathcal{T}^{2}=+1 \quad(1 \mathrm{D}$ class $\mathrm{AI})$. Examples include a onedimensional lattice with two sites per unit cell (Fig. 3a):

$$
\begin{aligned}
\hat{H}_{\mathrm{NHTI}}=\sum_{j} & \left\{\mathrm{i} t\left(\hat{a}_{j-1}^{\dagger} \hat{a}_{j}-\hat{b}_{j-1}^{\dagger} \hat{b}_{j}+\hat{a}_{j}^{\dagger} \hat{a}_{j-1}-\hat{b}_{j}^{\dagger} \hat{b}_{j-1}\right)\right. \\
& +\left[\mathrm{i} \delta\left(\hat{b}_{j-1}^{\dagger} \hat{a}_{j}-\hat{b}_{j+1}^{\dagger} \hat{a}_{j}\right)+\mathrm{i} \delta^{*}\left(\hat{a}_{j}^{\dagger} \hat{b}_{j-1}-\hat{a}_{j}^{\dagger} \hat{b}_{j+1}\right)\right] \\
& \left.+\mathrm{i} \gamma\left(\hat{a}_{j}^{\dagger} \hat{a}_{j}-\hat{b}_{j}^{\dagger} \hat{b}_{j}\right)\right\},
\end{aligned}
$$

where $\hat{a}_{j}\left(\hat{a}_{j}^{\dagger}\right)$ and $\hat{b}_{j}\left(\hat{b}_{j}^{\dagger}\right)$ denote the annihilation (creation) operators on each sublattice site $j, t>0$ and $\delta \in \mathbb{C}$ are the asymmetric-hopping amplitudes, and $\gamma \in \mathbb{R}$ is the balanced gain and loss. Such gain and loss have been experimentally implemented in various systems ${ }^{36-42,57-66}$ and the asymmetric hopping in optical systems ${ }^{67}$. The system respects time-reversal symmetry $\left(\hat{\mathcal{T}} \hat{H}_{\mathrm{NHTI}} \hat{\mathcal{T}}^{-1}=\hat{H}\right)$, where the time-reversal operation is defined by $\hat{\mathcal{T}}_{a_{j}} \hat{\mathcal{T}}^{-1}=\hat{b}_{j}, \hat{\mathcal{T}} \hat{b}_{j} \hat{\mathcal{T}}^{-1}=\hat{a}_{j}$, and $\hat{\mathcal{T}} z \hat{\mathcal{T}}^{-1}=z^{*}$ for all $z \in \mathbb{C}$. The eigenstates form two bands in momentum space, for which the Hamiltonian is determined as $\vec{h}(k) \cdot \vec{\sigma}$ with $h_{x}=-2 \mathrm{i} \operatorname{Im}[\delta] \sin k, h_{y}==2 \mathrm{i} \operatorname{Re}[\delta] \sin k, h_{z}=\mathrm{i}(\gamma+2 t \cos k)$, and Pauli matrices $\vec{\sigma}:=\left(\sigma_{x}, \sigma_{y}, \sigma_{z}\right)$. The energy dispersion is obtained as $E_{ \pm}(k)= \pm \mathrm{i} \sqrt{(\gamma+2 t \cos k)^{2}+4|\delta|^{2} \sin ^{2} k}$, and hence the complex bands are separated from each other by the energy gap with magnitude min $\{2|\gamma+2 t|, 2|\gamma-2 t|\}$ (Fig. 3b).

In parallel with the Majorana chain ${ }^{14}$, the topological invariant $\nu_{\text {AI }}$ is defined by

$$
(-1)^{\nu_{\mathrm{AI}}}:=\operatorname{sgn}\left[\mathrm{i} h_{z}(0) \cdot \mathrm{i} h_{z}(\pi)\right]=-\operatorname{sgn}\left[\gamma^{2}-4 t^{2}\right] .
$$

As a hallmark of the non-Hermitian topological phase, a pair of edge states with zero imaginary energy appears when the bulk has non-trivial topology $\left(v_{\mathrm{AI}}=1\right.$; Fig. $\left.3 \mathrm{c}\right)$. Whereas the bulk states that belong to the band $E_{+}\left(E_{-}\right)$are amplified (attenuated) with time, the mid-gap edge states are topologically protected from such amplification and attenuation. In the case of $t=\delta$, for instance, the topologically protected edge states are obtained as

$$
\begin{gathered}
\hat{\Psi}_{\text {edge }}^{(\text {left })} \propto \mathrm{i} \sum_{j=1}^{L}\left(-\frac{\gamma}{2 t}\right)^{j-1}\left(\hat{a}_{j}-\hat{b}_{j}\right), \\
\hat{\Psi}_{\text {edge }}^{\text {(right) }} \propto \sum_{j=1}^{L}\left(-\frac{\gamma}{2 t}\right)^{j-1}\left(\hat{a}_{L-j+1}+\hat{b}_{L-j+1}\right),
\end{gathered}
$$

which satisfy $\left\|\left[\hat{H}_{\mathrm{NHTI}}, \hat{\Psi}_{\text {edge }}\right]\right\|=O\left(e^{-L / \xi}\right)$ with the localization length $\xi:=-(\log |\gamma / 2 t|)^{-1}$. These edge states are immune to 

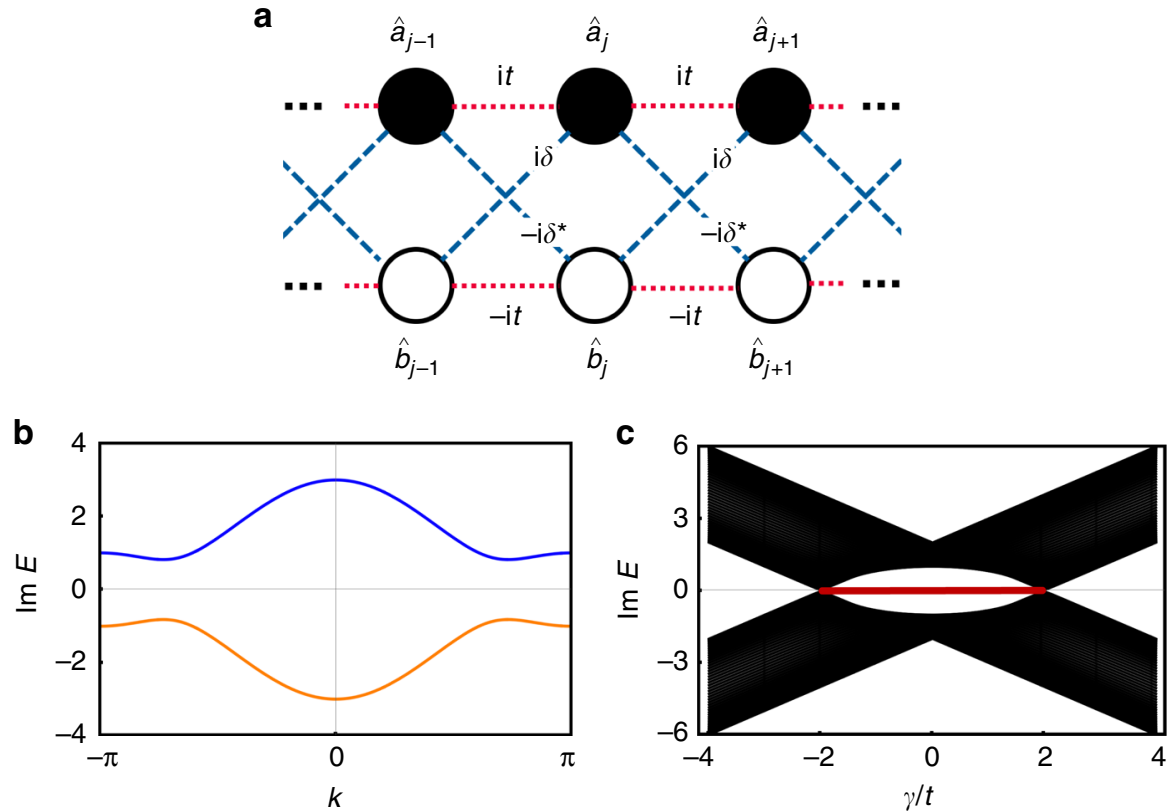

Fig. 3 Non-Hermitian one-dimensional topological insulator with time-reversal symmetry. The results shown are for the 1D class Al model described by Eq. (3). a Schematic representation of the Hamiltonian. The system consists of the asymmetric hopping and the gain/loss. $\mathbf{b}$ Energy dispersion of the chain with periodic boundaries $(t=1.0, \delta=0.5, \gamma=1.0)$. The imaginary spectrum is gapped for $|\gamma| \neq 2 t$. c Imaginary spectrum of the system of $L=50$ sites with open boundaries $(t=1.0, \delta=0.5)$. Edge states with $\operatorname{ImE}=0$ (red line) emerge in the topological phase $(|\gamma / t| \leq 2)$

disorder that respects time-reversal symmetry (see Supplementary Note 7 for details), which is a signature of the topological phase. We emphasize that topological phases are absent in 1D class $\mathrm{AI}$ in the presence of Hermiticity ${ }^{3-5}$; non-Hermiticity induces the unique non-equilibrium topological phase as a result of the topological unification of time-reversal and particle-hole symmetries. Whereas the system is an insulator and does not support non-Abelian Majorana fermions, the sublattice degrees of freedom $\hat{a}_{j}$ and $\hat{b}_{j}$ play the roles of particles and holes in the Majorana chain; the Majorana edge states, which are equalsuperposition states of particles and holes, correspond to the equal superposition states of the two sublattices $\hat{a}_{j}$ and $\hat{b}_{j}$ in the non-Hermitian topological insulator.

Emergent non-Hermitian topological phases. The topological phases induced by non-Hermiticity are not specific to the above model but general for all the non-Hermitian systems with antiunitary symmetry. To see this, we examine the complex-band structure of a generic two-band system $\left(E_{+}(k), E_{-}(k)\right)$ in 1D class AI. In the presence of Hermiticity, the real bands individually respect time-reversal symmetry: $E_{+}(k)=E_{+}^{*}(-k)$ (Fig. 4a), where topological phases are absent ${ }^{3,5}$. In the presence of strong non-Hermiticity, on the other hand, time-reversal symmetry is spontaneously broken and the complex bands are paired via time-reversal symmetry: $E_{+}(k)=E_{-}^{*}(-k)$. Importantly, this system has the same band structure as the Hermitian topological superconductor protected by particle-hole symmetry (1D class D) as a direct consequence of the topological unification of time-reversal and particle-hole symmetries; it exhibits both trivial (Fig. 4b) and topological (Fig. 4c) phases according to the $\mathbb{Z}_{2}$ topological invariant defined by Eq. (4). The latter band structure becomes gapless in the presence of Hermiticity due to $E_{+}\left(k_{0}\right)=E_{-}\left(k_{0}\right)$ for a time-reversal-invariant momentum $k_{0} \in$ $\{0, \pi\}$.

Remarkably, the emergent non-Hermitian topological phases cannot be continuously deformed into any Hermitian phase that belongs to the same symmetry class. In fact, there should exist a
non-Hermitian Hamiltonian that satisfies $E_{+}(k)=E_{-}(-k)$ between the two types of band structures, and the complex gap closes at $k=k_{0}$. Thus complex-gap closing associated with a topological phase transition should occur between these phases. We also emphasize that the above discussions are applicable to all the non-Hermitian topological phases in any spatial dimension protected by anti-unitary symmetry. Here the corresponding topological invariants are solely determined by the relationship between symmetry and the complex-band structure as in the Hermitian case $^{22}$.

Quantum spin Hall insulator. Topological phases survive nonHermiticity also in two dimensions. In fact, the $\mathbb{Z}_{2}$ topological invariant $\nu_{\mathrm{AII}}$ can be defined in non-Hermitian two-dimensional insulators that respect both time-reversal and parity (inversion) symmetries just like the Hermitian ones ${ }^{11}$ :

$$
(-1)^{\nu_{\text {AII }}}:=\prod_{\boldsymbol{k}_{0}} \prod_{n: \text { occupied }} \pi_{n}\left(\boldsymbol{k}_{0}\right)
$$

where $\boldsymbol{k}_{0} \in\{(0,0),(0, \pi),(\pi, 0),(\pi, \pi)\}$ denotes the time-reversalinvariant and inversion-symmetric momenta in the Brillouin zone, and $\pi_{n}\left(\boldsymbol{k}_{0}\right) \in\{ \pm 1\}$ is the parity eigenvalue of the $n$-th Kramers pair at $\boldsymbol{k}=\boldsymbol{k}_{0}$. In particular, for four-band insulators such as the Kane-Mele model ${ }^{8}$ and the Bernevig-Hughes-Zhang

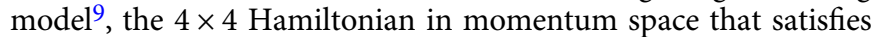
$\mathcal{T} \mathcal{H}(\boldsymbol{k}) \mathcal{T}^{-1}=\mathcal{H}(-\boldsymbol{k})$ and $\mathcal{P} \mathcal{H}(\boldsymbol{k}) \mathcal{P}^{-1}=\mathcal{H}(-\boldsymbol{k})$ is expressed as

$$
\mathcal{H}_{\mathrm{QSH}}(\boldsymbol{k})=d_{0}(\boldsymbol{k}) I+\vec{d}(\boldsymbol{k}) \cdot \vec{\Gamma}+\mathrm{i} \sum_{1 \leq i<j \leq 5} d_{i j}(\boldsymbol{k}) \Gamma_{i j},
$$

where the coefficients $d_{i}$ 's and $d_{i j}$ 's are real, $\Gamma_{I}$ 's are $\mathcal{P} \mathcal{T}$-symmetric five Dirac matrices, and $\Gamma_{i j}$ 's are their commutators $\Gamma_{i j}$ : $=$ $\left[\Gamma_{i}, \Gamma_{j}\right] / 2$ i. We notice that Hermiticity leads to $d_{i j}=0$. Here only $\Gamma_{1}$ and $\Gamma_{i j}(2 \leq i<j \leq 5)$ are invariant under space inversion when $\Gamma_{1}$ is chosen as $\mathcal{P}^{11}$. Moreover, when the parity and time-reversal operators are given as $\mathcal{P}=\sigma_{z}$ and $\mathcal{T}=\mathrm{i} s_{y} \mathcal{K}$, the Dirac matrices can be expressed as $\Gamma_{1}=\sigma_{z}(=\mathcal{P}), \Gamma_{2}=\sigma_{y}$, 


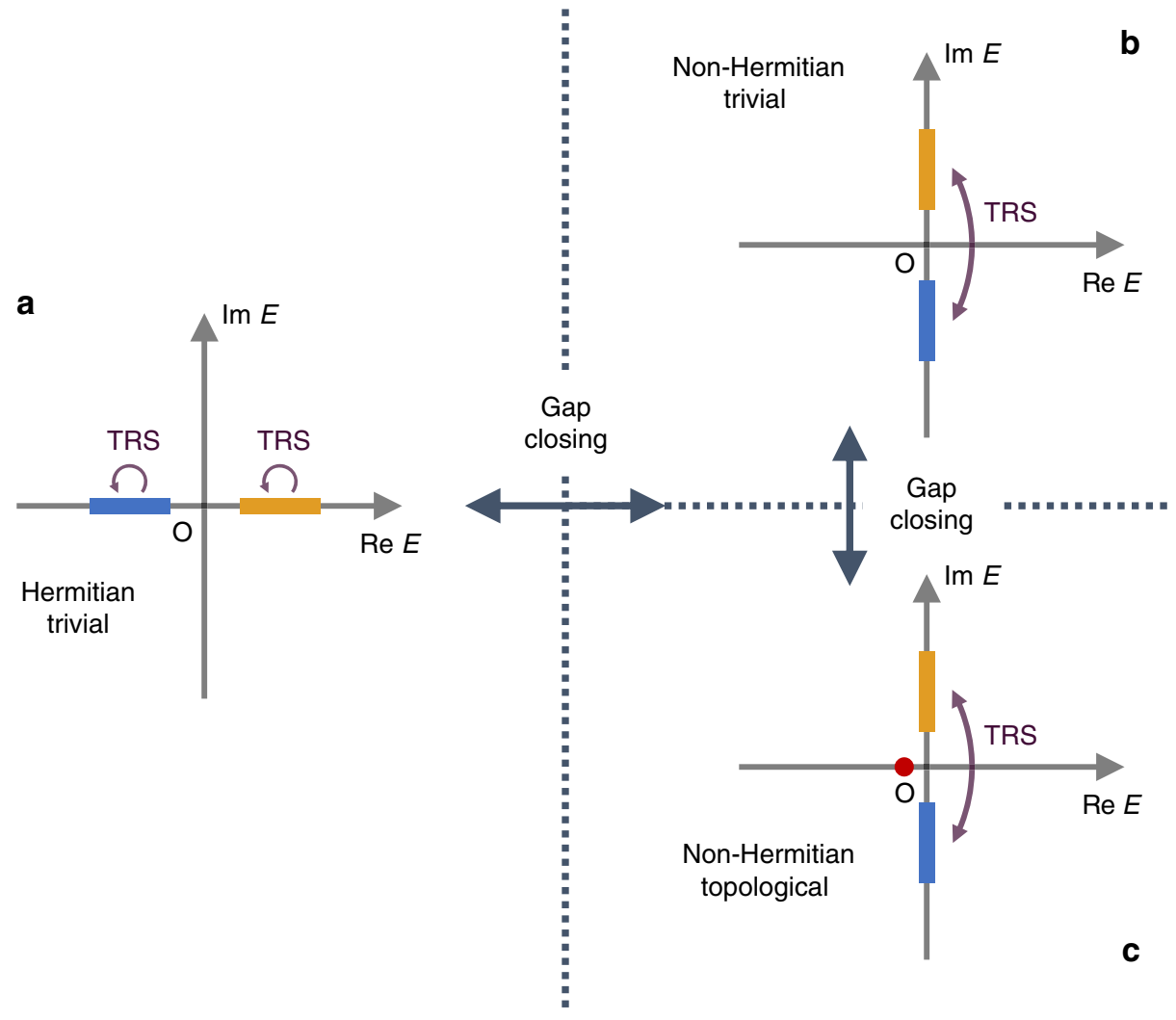

Fig. 4 Complex-band structure of non-Hermitian one-dimensional insulators with time-reversal symmetry (1D class Al). Blue and yellow bands represent complex bands and red dots represent topologically protected edge states. a Hermitian gapped band structure. All the bands individually respect timereversal symmetry, and topological phases are absent. b, c Non-Hermitian gapped band structure in 1D class Al for $\mathbf{b}$ the trivial phase and $\mathbf{c}$ the topological phase. Two bands are paired via time-reversal symmetry and the complex-gap closing associated with a topological phase transition should occur between (a) and (b, c)

$\Gamma_{3}=\sigma_{x} s_{x}, \Gamma_{4}=\sigma_{x} s_{y}$, and $\Gamma_{5}=\sigma_{x} s_{z}{ }^{11}$. Here $\sigma_{i}^{\prime}$ s $\left(s_{i}^{\prime} s\right)$ denote the Pauli matrices that describe the sublattice (spin) degrees of freedom. Since the Hamiltonian at $\boldsymbol{k}=\boldsymbol{k}_{0}$ is invariant under inversion $\mathcal{P} \mathcal{H}\left(\boldsymbol{k}_{0}\right) \mathcal{P}^{-1}=\mathcal{H}\left(\boldsymbol{k}_{0}\right)$, it reduces to $\mathcal{H}_{\mathrm{QSH}}\left(\boldsymbol{k}_{0}\right)=d_{0}\left(\boldsymbol{k}_{0}\right) I+d_{1}\left(\boldsymbol{k}_{0}\right) \mathcal{P}+\mathrm{i} \sum_{1 \leq i<j \leq 5} d_{i j}\left(\boldsymbol{k}_{0}\right) \Gamma_{i j}$; the parity of a Kramers pair at $\boldsymbol{k}=\boldsymbol{k}_{0}$ corresponds to the sign of $d_{1}\left(\boldsymbol{k}_{0}\right)$, and the $\mathbb{Z}_{2}$ topological invariant defined by Eq. (6) is obtained as $(-1)^{\nu_{\mathrm{AII}}^{2}}=\prod_{\boldsymbol{k}_{0}} \operatorname{sgn}\left[d_{1}\left(\boldsymbol{k}_{0}\right)\right]$ as long as complex bands are gapped and $d_{1}\left(\boldsymbol{k}_{0}\right)$ is non-zero.

This bulk $\mathbb{Z}_{2}$ topological invariant corresponds to the emergence of helical edge states (Fig. 5). In stark contrast to Hermitian systems $^{8-11}$, the helical edge states form not a Dirac point but a pair of exceptional points ${ }^{30,31,40,63,69,70}$ and have non-zero imaginary energies at the time-reversal-invariant momenta. Nevertheless, they are immune to disorder due to the generalized Kramers theorem (see Supplementary Notes 5 and 7 for details), which states that all the real parts of energies should be degenerate in the presence of time-reversal symmetry with $\mathcal{T}^{2}=-1$; the degeneracies of the real parts of energies forbid the continuous annihilation of a pair of helical edge states. Notably, the helical edge states are lasing (see Supplementary Note 8 for details) like chiral edge states in a nonHermitian Chern insulator ${ }^{42}$.

The topological unification of anti-unitary symmetries indicates that non-Hermitian systems that respect particle-hole symmetry with $\mathcal{C}^{2}=-1 \quad(2 \mathrm{D}$ class $\mathrm{C})$ also exhibit the $\mathbb{Z}_{2}$ topological phase, in contrast to the $2 \mathbb{Z}$ topological phase in Hermitian physics ${ }^{3-5}$. Here the spin-up and spin-down particles in insulators correspond to particles and holes in superconductors. This emergent $\mathbb{Z}_{2}$ topological phase is due to the presence of Kramers pairs of particles and holes with imaginary energies, which are forbidden in Hermitian systems where energies are confined to the real axis; non-Hermiticity brings about topological phases unique to non-equilibrium open systems.

\section{Discussion}

Non-Hermiticity manifests itself in many disciplines of physics as gain and loss or asymmetric hopping ${ }^{43,44}$. We have shown that such non-Hermiticity unifies the two fundamental anti-unitary symmetries and consequently topological classification, leading to the prediction of unique non-equilibrium topological phases that are absent at equilibrium. The unveiled topological unification of time-reversal and particle-hole symmetries provides a general symmetry principle in non-Hermitian physics that also justifies a different type of topological classification 68 . The modified topological classification implies that the symmetry unification can bring about physics unique to non-Hermitian systems. It merits further study to explore such unusual properties and functionalities that result from our symmetry principle.

This work has explored topological phases characterized by wave functions in non-Hermitian gapped systems, which is a non-trivial generalization of the Hermitian topological phases. By contrast, non-Hermitian gapless systems possess an intrinsic topological structure, which accompanies exceptional points ${ }^{69,70}$, and has no counterparts in Hermitian systems. This topology can be characterized by a complex-energy dispersion $28,30,31$ and is distinct from the topology defined by wave functions. A complete theory of non-Hermitian topological systems should be formulated on the basis of these two types of topology in a unified manner, which awaits further theoretical development. 

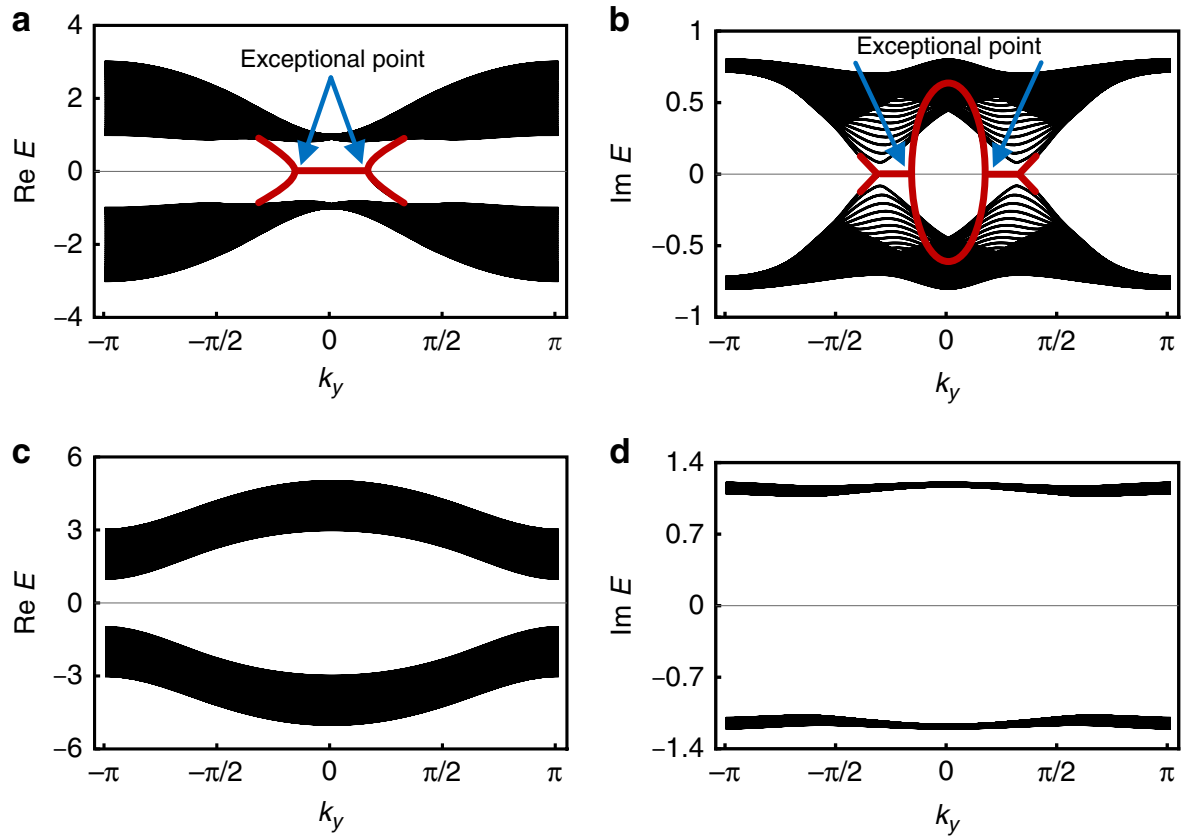

Fig. 5 Non-Hermitian quantum spin Hall insulator. The results shown are for the model described by Eq. (7) with $d_{1}(\boldsymbol{k})=m+t \cos k_{x}+t \cos k_{y}, d_{2}(\boldsymbol{k})=t$ $\sin k_{y}, d_{3}(\boldsymbol{k})=\lambda\left(\sin k_{x}+\sin k_{y}\right), d_{5}(\boldsymbol{k})=t \sin k_{x}$ and $d_{25}(\boldsymbol{k})=\gamma(t, m, \lambda, \gamma \in \mathbb{R})$. We here consider a non-Hermitian two-dimensional insulator on a square lattice with open boundaries in the $x$ direction and periodic boundaries in the $y$ direction, along which the wavenumber $k_{y}$ is well-defined. The $\mathbb{Z}_{2}$ topological invariant is given by $(-1)^{\nu \text { AII }}=\operatorname{sgn}\left[m^{2}-4 t^{2}\right]$. a Real and $\mathbf{b}$ imaginary parts of the complex spectrum in the topological phase $(t=1.0, m=-1.0, \lambda=0.5, \gamma=$ $0.8 ; \nu_{\text {AII }}=1$ ). Helical edge states (red curves) appear between the gapped complex bands and form a pair of exceptional points. $\mathbf{c}$ Real and $\mathbf{d}$ imaginary parts of the complex spectrum in the trivial phase $\left(t=1.0, m=3.0, \lambda=0.8, \gamma=1.2 ; \nu_{\text {AII }}=0\right)$. No gapless states appear between the gapped complex bands

\section{Data availability}

The data that support the findings of this study are available from the corresponding author on reasonable request.

Received: 17 November 2018 Accepted: 21 December 2018

Published online: 17 January 2019

\section{References}

1. Wigner, E. P. Group Theory and its Application to the Quantum Mechanics of Atomic Spectra (Academic Press, New York, 1959).

2. Altland, A. \& Zirnbauer, M. R. Nonstandard symmetry classes in mesoscopic normal-superconducting hybrid structures. Phys. Rev. B 55, 1142-1161 (1997).

3. Hasan, M. Z. \& Kane, C. L. Colloquium: topological insulators. Rev. Mod. Phys. 82, 3045-3067 (2010).

4. Qi, X.-L. \& Zhang, S.-C. Topological insulators and superconductors. Rev. Mod. Phys. 83, 1057-1110 (2011).

5. Chiu, C.-K., Teo, J. C. Y., Schnyder, A. P. \& Ryu, S. Classification of topological quantum matter with symmetries. Rev. Mod. Phys. 88, 035005 (2016).

6. Thouless, D. J., Kohmoto, M., Nightingale, M. P. \& den Nijs, M. Quantized Hall conductance in a two-dimensional periodic potential. Phys. Rev. Lett. 49, 405-408 (1982).

7. Haldane, F. D. M. Model for a quantum Hall effect without Landau levels: condensed-matter realization of the "parity anomaly". Phys. Rev. Lett. 61, 2015-2018 (1988).

8. Kane, C. L. \& Mele, E. J. $Z_{2}$ topological order and the quantum spin Hall effect. Phys. Rev. Lett. 95, 146802 (2005).

9. Bernevig, B. A., Hughes, T. L. \& Zhang, S.-C. Quantum spin Hall effect and topological phase transition in HgTe quantum wells. Science 314, 1757-1761 (2006).

10. Moore, J. E. \& Balents, L. Topological invariants of time-reversal-invariant band structures. Phys. Rev. B 75, 121306(R) (2007).

11. Fu, L. \& Kane, C. L. Topological insulators with inversion symmetry. Phys. Rev. B 76, 045302 (2007).
12. König, M. et al. Quantum spin Hall insulator state in $\mathrm{HgTe}$ quantum wells. Science 318, 766-770 (2007).

13. Read, N. \& Green, D. Paired states of fermions in two dimensions with breaking of parity and time-reversal symmetries and the fractional quantum Hall effect. Phys. Rev. B 61, 10267-10297 (2000).

14. Kitaev, A. Y. Unpaired Majorana fermions in quantum wires. Phys. -Usp. 44, 131-136 (2001).

15. Ivanov, D. A. Non-Abelian statistics of half-quantum vortices in $p$-wave superconductors. Phys. Rev. Lett. 86, 268-271 (2001).

16. Fu, L. \& Kane, C. L. Superconducting proximity effect and Majorana fermions at the surface of a topological insulator. Phys. Rev. Lett. 100, 096407 (2008).

17. Lu, L., Joannopoulos, J. D. \& Soljačić, M. Topological photonics. Nat. Photonics. 8, 821-829 (2014).

18. Goldman, N., Budich, J. C. \& Zoller, P. Topological quantum matter with ultracold gases in optical lattices. Nat. Phys. 12, 639-645 (2016).

19. Schnyder, A. P., Ryu, S., Furusaki, A. \& Ludwig, A. W. W. Classification of topological insulators and superconductors in three spatial dimensions. Phys. Rev. B 78, 195125 (2008).

20. Kitaev, A. Periodic table for topological insulators and superconductors. AIP Conf. Proc. 1134, 22-30 (2009).

21. Ryu, S., Schnyder, A. P., Furusaki, A. \& Ludwig, A. W. W. Topological insulators and superconductors: tenfold way and dimensional hierarchy. New J. Phys. 12, 065010 (2010)

22. Teo, J. C. Y. \& Kane, C. L. Topological defects and gapless modes in insulators and superconductors. Phys. Rev. B 82, 115120 (2010).

23. Rudner, M. S. \& Levitov, L. S. Topological transition in a non-Hermitian quantum walk. Phys. Rev. Lett. 102, 065703 (2009).

24. Hu, Y. C. \& Hughes, T. L. Absence of topological insulator phases in non Hermitian PT-symmetric Hamiltonians. Phys. Rev. B 84, 153101 (2011).

25. Esaki, K., Sato, M., Hasebe, K. \& Kohmoto, M. Edge states and topological phases in non-Hermitian systems. Phys. Rev. B 84, 205128 (2011).

26. Malzard, S., Poli, C. \& Schomerus, H. Topologically protected defect states in open photonic systems with non-Hermitian charge-conjugation and paritytime symmetry. Phys. Rev. Lett. 115, 200402 (2015).

27. San-Jose, P., Carao, J., Prada, E. \& Aguado, R. Majorana bound states from exceptional points in non-topological superconductors. Sci. Rep. 6, 21427 (2016).

28. Lee, T. E. Anomalous edge state in a non-Hermitian lattice. Phys. Rev. Lett. 116, 133903 (2016). 
29. Leykam, D., Bliokh, K. Y., Huang, C., Chong, Y. D. \& Nori, F. Edge modes, degeneracies, and topological numbers in non-Hermitian systems. Phys. Rev. Lett. 118, 040401 (2017).

30. Xu, Y., Wang, S.-T. \& Duan, L.-M. Weyl exceptional rings in a threedimensional dissipative cold atomic gas. Phys. Rev. Lett. 118, 045701 (2017).

31. Shen, H., Zhen, B. \& Fu, L. Topological band theory for non-Hermitian Hamiltonians. Phys. Rev. Lett. 120, 146402 (2018).

32. Kunst, F. K., Edvardsson, E., Budich, J. C. \& Bergholtz, E. J. Biorthogonal bulk-boundary correspondence in non-Hermitian systems. Phys. Rev. Lett. 121, 026808 (2018).

33. Kawabata, K., Ashida, Y., Katsura, H. \& Ueda, M. Parity-time-symmetric topological superconductor. Phys. Rev. B 98, 085116 (2018).

34. Yao, S. \& Wang, Z. Edge states and topological invariants of non-Hermitian systems. Phys. Rev. Lett. 121, 086803 (2018).

35. Kozii, V. \& Fu, L. Non-Hermitian topological theory of finite-lifetime quasiparticles: prediction of bulk Fermi arc due to exceptional point. Preprint at https://arxiv.org/abs/1708.05841 (2017).

36. Zeuner, J. M. et al. Observation of a topological transition in the bulk of a nonHermitian system. Phys. Rev. Lett. 115, 040402 (2015).

37. Weimann, S. et al. Topologically protected bound states in photonic paritytime-symmetric crystals. Nat. Mater. 16, 433-438 (2017).

38. Xiao, L. et al. Observation of topological edge states in parity-time-symmetric quantum walks. Nat. Phys. 13, 1117-1123 (2017).

39. St-Jean, P. et al. Lasing in topological edge states of a one-dimensional lattice. Nat. Photonics. 11, 651-656 (2017).

40. Zhou, H. et al. Observation of bulk Fermi arc and polarization half charge from paired exceptional points. Science 359, 1009-1012 (2018).

41. Parto, M. et al. Edge-mode lasing in $1 \mathrm{D}$ topological active arrays. Phys. Rev. Lett. 120, 113901 (2018).

42. Bandres, M. A. et al. Topological insulator laser: experiments. Science 359, eaar4005 (2018).

43. Konotop, V. V., Yang, J. \& Zezyulin, D. A. Nonlinear waves in PT-symmetric systems. Rev. Mod. Phys. 88, 035002 (2016).

44. El-Ganainy, R. et al. Non-Hermitian physics and PT symmetry. Nat. Phys. 14, 11-19 (2018)

45. Hatano, N. \& Nelson, D. R. Localization transitions in non-Hermitian quantum mechanics. Phys. Rev. Lett. 77, 570-573 (1996).

46. Bender, C. M. \& Boettcher, S. Real spectra in non-Hermitian Hamiltonians having PT symmetry. Phys. Rev. Lett. 80, 5243-5246 (1998).

47. Bender, C. M., Brody, D. C., Jones, H. F. \& Meister, B. K. Faster than Hermitian quantum mechanics. Phys. Rev. Lett. 98, 040403 (2007).

48. Makris, K. G., El-Ganainy, R., Christodoulides, D. N. \& Musslimani, Z. H. Beam dynamics in PT symmetric optical lattices. Phys. Rev. Lett. 100, 103904 (2008).

49. Klaiman, S., Günther, U. \& Moiseyev, N. Visualization of branch points in PTsymmetric waveguides. Phys. Rev. Lett. 101, 080402 (2008).

50. Lin, Z. et al. Unidirectional invisibility induced by PT-symmetric periodic structures. Phys. Rev. Lett. 106, 213901 (2011).

51. Brody, D. C. \& Graefe, E.-M. Mixed-state evolution in the presence of gain and loss. Phys. Rev. Lett. 109, 230405 (2012).

52. Ge, L. Symmetry-protected zero-mode laser with a tunable spatial profile. Phys. Rev. A. 95, 023812 (2017).

53. Ashida, Y., Furukawa, S. \& Ueda, M. Parity-time-symmetric quantum critical phenomena. Nat. Commun. 8, 15791 (2017).

54. Kawabata, K., Ashida, Y. \& Ueda, M. Information retrieval and criticality in parity-time-symmetric systems. Phys. Rev. Lett. 119, 190401 (2017).

55. Lau, H.-K. \& Clerk, A. A. Fundamental limits and non-reciprocal approaches in non-Hermitian quantum sensing. Nat. Commun. 9, 4320 (2018).

56. Nakagawa, M., Kawakami, N. \& Ueda, M. Non-Hermitian Kondo effect in ultracold alkaline-earth atoms. Phys. Rev. Lett. 121, 203001 (2018).

57. Guo, A. et al. Observation of PT-symmetry breaking in complex optical potentials. Phys. Rev. Lett. 103, 093902 (2009).

58. Rüter, C. E. et al. Observation of parity-time symmetry in optics. Nat. Phys. 6, 192-195 (2010).

59. Regensburger, A. et al. Parity-time synthetic photonic lattices. Nature 488, 167-171 (2012).

60. Peng, B. et al. Parity-time-symmetric whispering-gallery microcavities. Nat. Phys. 10, 394-398 (2014).

61. Feng, L., Wong, Z. J., Ma, R.-M., Wang, Y. \& Zhang, X. Single-mode laser by parity-time symmetry breaking. Science 346, 972-975 (2014).
62. Hodaei, H. et al. Parity-time-symmetric microring lasers. Science 346, 975-978 (2014).

63. Zhen, B. et al. Spawning rings of exceptional points out of Dirac cones. Nature 525, 354-358 (2015).

64. Gao, T. et al. Observation of non-Hermitian degeneracies in a chaotic excitonpolariton billiard. Nature 526, 554-558 (2015).

65. Peng, P. et al. Anti-parity-time symmetry with flying atoms. Nat. Phys. 12, 1139-1145 (2016)

66. Hodaei, H. et al. Enhanced sensitivity at higher-order exceptional points. Nature 548, 187-191 (2017).

67. Chen, W. et al. Exceptional points enhance sensing in an optical microcavity. Nature 548, 192-196 (2017).

68. Gong, Z. et al. Topological phases of non-Hermitian systems. Phys. Rev. X 8, 031079 (2018)

69. Berry, M. V. Physics of nonhermitian degeneracies. Czech. J. Phys. 54, 1039-1047 (2004)

70. Heiss, W. D. The physics of exceptional points. J. Phys. A 45, 444016 (2012).

\section{Acknowledgements}

K.K. thanks Aashish A. Clerk, Li Ge, Hosho Katsura, Masatoshi Sato, Ken Shiozaki, Zhong Wang, and Haruki Watanabe for helpful discussions. This work was supported by KAKENHI Grant No. JP18H01145 and a Grant-in-Aid for Scientific Research on Innovative Areas "Topological Materials Science" (KAKENHI Grant No. JP15H05855) from the Japan Society for the Promotion of Science (JSPS). K.K., S.H., and Y.A. were supported by the JSPS through Program for Leading Graduate Schools (ALPS). S.H. and Y.A. acknowledge the support from JSPS (KAKENHI Grant Nos. JP16J03619 and JP16J03613). Z.G. acknowledges the support from MEXT.

\section{Author contributions}

K.K. conceived the main idea, and K.K. and S.H. proved it. K.K. investigated the illustrative examples and generalized them. M.U. supervised the work. K.K., S.H., Z.G., Y.A., and M.U. extensively analyzed and interpreted the results and contributed to the writing of the manuscript.

\section{Additional information}

Supplementary Information accompanies this paper at https://doi.org/10.1038/s41467018-08254-y.

Competing interests: The authors declare no competing interests.

Reprints and permission information is available online at http://npg.nature.com/ reprintsandpermissions/

Journal peer review information: Nature Communications thanks the anonymous reviewers for their contribution to the peer review of this work. Peer reviewer reports are available.

Publisher's note: Springer Nature remains neutral with regard to jurisdictional claims in published maps and institutional affiliations.

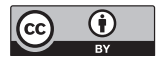

Open Access This article is licensed under a Creative Commons Attribution 4.0 International License, which permits use, sharing, adaptation, distribution and reproduction in any medium or format, as long as you give appropriate credit to the original author(s) and the source, provide a link to the Creative Commons license, and indicate if changes were made. The images or other third party material in this article are included in the article's Creative Commons license, unless indicated otherwise in a credit line to the material. If material is not included in the article's Creative Commons license and your intended use is not permitted by statutory regulation or exceeds the permitted use, you will need to obtain permission directly from the copyright holder. To view a copy of this license, visit http://creativecommons.org/ licenses/by/4.0/.

(C) The Author(s) 2019 